Article

\title{
Development of a new numerical scheme for the solution of exponential growth and decay models
}

\section{S. E. Fadugba}

Department of Mathematics, Faculty of Science, Ekiti State University, Ado Ekiti, Nigeria.; sunday.fadugba@eksu.edu.ng Academic Editor: Wei Gao

Received: 28 April 2020; Accepted: 25 December 2020; Published: 19 January 2021.

\begin{abstract}
This paper presents the development of a new numerical scheme for the solution of exponential growth and decay models emanated from biological sciences. The scheme has been derived via the combination of two interpolants namely, polynomial and exponential functions. The analysis of the local truncation error of the derived scheme is investigated by means of the Taylor's series expansion. In order to test the performance of the scheme in terms of accuracy in the context of the exact solution, four biological models were solved numerically. The absolute error has been computed successfully at each mesh point of the integration interval under consideration. The numerical results generated via the scheme agree with the exact solution and with the fifth order convergence based upon the analysis carried out. Hence, the scheme is found to be of order five, accurate and is a good approach to be included in the class of linear explicit numerical methods for the solution of initial value problems in ordinary differential equations.
\end{abstract}

Keywords: Accuracy, biological model, ordinary differential equation, initial value problem, local truncation error.

MSC: 34A12, 65L05, 65L70.

\section{Introduction}

D ifferential equations are useful to modern science and engineering. Differential equation models are used extensively in biology to study biochemical reactions, population dynamics, organism growth, and the spread of diseases. The most common use of differential equations in science is to model dynamical systems. Such physical models represent future estimation for any real world situation based on the data available in the past and present as detailed in [1-9].

However, these models are said to have no closed form solution in most of the real cases. In such situations, one has to compromise at numerical approximate solutions of the models achievable by various numerical techniques of different characteristics [10]. Development of new numerical integration methods with varying characteristics for the solution of initial value problems in ordinary differential equations has attracted the attention of many researchers in past and recent years as detailed in [11-17].

The main aim of the paper is to develop a new numerical method of order five via the combination of two interpolants for its possible acceptance within the class of linear explicit numerical techniques. Also the local truncation errors and order of accuracy of the scheme were thoroughly investigated. The rest of the paper is structured as follows; Section 2 presents the development of the scheme. In Section 3, the local truncation error of the scheme has been investigated. Also the order of accuracy of the scheme is obtained. Section 4 presents numerical experiments, discussion of results and concluding remarks.

\section{Development of a new fifth order scheme}

Consider an interpolating function of the form

$$
F(x)=\sum_{j=0}^{5} \beta_{j} x^{j}+\beta_{6} e^{c},
$$


where $\beta_{0}, \beta_{2}, \beta_{3}, \ldots, \beta_{6}$ are undetermined constants and $c$ is a constant. The integration interval of $[a, b]$ is defined as

$$
a=x_{0} \leq x \leq x_{n}=b
$$

The step length is defined as

$$
h=\frac{b-a}{N} .
$$

The mesh point is defined as

$$
x_{n}=x_{0}+n h, n=1,2, . ., N
$$

or

$$
x_{n+1}=x_{0}+(n+1) h, n=0,1,2, \ldots, N-1 .
$$

Expanding (1) at the points $x_{n}$ and $x_{n+1}$ yields

$$
F\left(x_{n}\right)=\sum_{j=1}^{5} \beta_{j} x_{n}^{j}+\beta_{6} e^{c},
$$

and

$$
F\left(x_{n+1}\right)=\sum_{j=1}^{5} \beta_{j} x_{n+1}^{j}+\beta_{6} e^{c}
$$

respectively. Differentiating (6) five times and using the fact that

$$
F^{\prime}\left(x_{n}\right)=f_{n}, F^{\prime \prime}\left(x_{n}\right)=f_{n}^{(1)}, F^{\prime \prime \prime}\left(x_{n}\right)=f_{n}^{(2)}, F^{(i v)}\left(x_{n}\right)=f_{n}^{(3)}, F^{(v)}\left(x_{n}\right)=f_{n}^{(4)}
$$

yields

$$
\begin{aligned}
f_{n} & =\sum_{j=1}^{5} j \beta_{j} x_{n}^{j-1}, \\
f_{n}^{(1)} & =\sum_{j=2}^{5} j(j-1) \beta_{j} x_{n}^{j-2}, \\
f_{n}^{(2)} & =\sum_{j=3}^{5} j(j-1)(j-2) \beta_{j} x_{n}^{j-3}, \\
f_{n}^{(3)} & =\sum_{j=4}^{5} j(j-1)(j-2)(j-3) \beta_{j} x_{n}^{j-4}, \\
f_{n}^{(4)} & =\sum_{j=5}^{5} j(j-1)(j-2)(j-3)(j-4) \beta_{j} x_{n}^{j-5} .
\end{aligned}
$$

Equations (8) - (12) form a system of linear equations $A X=b$ which can be represented in a matrix form as follows;

$$
\left[\begin{array}{ccccc}
1 & 2 x_{n} & 3 x_{n}^{2} & 4 x_{n}^{3} & 5 x_{n}^{4} \\
0 & 2 & 6 x_{n} & 12 x_{n}^{2} & 20 x_{n}^{3} \\
0 & 0 & 6 & 24 x_{n} & 60 x_{n}^{2} \\
0 & 0 & 0 & 24 & 120 x_{n} \\
0 & 0 & 0 & 0 & 120
\end{array}\right]\left[\begin{array}{l}
\beta_{1} \\
\beta_{2} \\
\beta_{3} \\
\beta_{4} \\
\beta_{5}
\end{array}\right]=\left[\begin{array}{c}
f_{n} \\
f_{n}^{(1)} \\
f_{n}^{(2)} \\
f_{n}^{(3)} \\
f_{n}^{(4)}
\end{array}\right]
$$

where

$$
A=\left[\begin{array}{ccccc}
1 & 2 x_{n} & 3 x_{n}^{2} & 4 x_{n}^{3} & 5 x_{n}^{4} \\
0 & 2 & 6 x_{n} & 12 x_{n}^{2} & 20 x_{n}^{3} \\
0 & 0 & 6 & 24 x_{n} & 60 x_{n}^{2} \\
0 & 0 & 0 & 24 & 120 x_{n} \\
0 & 0 & 0 & 0 & 120
\end{array}\right], X=\left[\begin{array}{c}
\beta_{1} \\
\beta_{2} \\
\beta_{3} \\
\beta_{4} \\
\beta_{5}
\end{array}\right], b=\left[\begin{array}{c}
f_{n} \\
f_{n}^{(1)} \\
f_{n}^{(2)} \\
f_{n}^{(3)} \\
f_{n}^{(4)}
\end{array}\right]
$$


Solving (13) by means of the Gauss Jordan method yields

$$
\begin{aligned}
& \beta_{1}=\frac{1}{24}\left(24 f_{n}-24 x_{n} f_{n}^{(1)}+12 x_{n}^{2} f_{n}^{(2)}-4 x_{n}^{3} f_{n}^{(3)}+x_{n}^{4} f_{n}^{(4)}\right) \\
& \beta_{2}=\frac{1}{12}\left(6 f_{n}^{(1)}-6 x_{n} f_{n}^{(2)}+3 x_{n}^{2} f_{n}^{(3)}-x_{n}^{3} f_{n}^{(4)}\right) \\
& \beta_{3}=\frac{1}{12}\left(2 f_{n}^{(2)}-2 x_{n} f_{n}^{(3)}+x_{n}^{2} f_{n}^{(4)}\right) \\
& \beta_{4}=\frac{1}{24}\left(f_{n}^{(3)}-x_{n} f_{n}^{(4)}\right) \\
& \beta_{5}=\frac{1}{120} f_{n}^{(4)}
\end{aligned}
$$

Subtracting (6) from (7) yields

$$
F\left(x_{n+1}\right)-F\left(x_{n}\right)=\sum_{j=1}^{5} \beta_{j}\left(x_{n+1}^{j}-x_{n}^{j}\right) .
$$

Therefore, (19) becomes

$$
F\left(x_{n+1}\right)-F\left(x_{n}\right)=\beta_{1}\left(x_{n+1}-x_{n}\right)+\beta_{2}\left(x_{n+1}^{2}-x_{n}^{2}\right)+\beta_{3}\left(x_{n+1}^{3}-x_{n}^{3}\right)+\beta_{4}\left(x_{n+1}^{4}-x_{n}^{4}\right)+\beta_{5}\left(x_{n+1}^{5}-x_{n}^{5}\right) .
$$

Using (4) and (5) with $x_{0}=0$, one obtains the following

$$
\begin{aligned}
& x_{n}=n h, \\
& x_{n+1}=(n+1) h, \\
& x_{n+1}-x_{n}=h, \\
& x_{n+1}^{2}-x_{n}^{2}=(2 n+1) h^{2}, \\
& x_{n+1}^{3}-x_{n}^{3}=\left(3 n^{2}+3 n+1\right) h^{3}, \\
& x_{n+1}^{4}-x_{n}^{4}=\left(4 n^{3}+6 n^{2}+4 n+1\right) h^{4}, \\
& x_{n+1}^{5}-x_{n}^{5}=\left(5 n^{4}+10 n^{3}+10 n^{2}+5 n+1\right) h^{5} .
\end{aligned}
$$

Using (21), then (14), (15), (16), (17) and (18) become

$$
\begin{aligned}
& \beta_{1}=\frac{1}{24}\left(24 f_{n}-24 n h f_{n}^{(1)}+12 n^{2} h^{2} f_{n}^{(2)}-4 n^{3} h^{3} f_{n}^{(3)}+n^{4} h^{4} f_{n}^{(4)}\right), \\
& \beta_{2}=\frac{1}{12}\left(6 f_{n}^{(1)}-6 n h f_{n}^{(2)}+3 n^{2} h^{2} f_{n}^{(3)}-n^{3} h^{3} f_{n}^{(4)}\right), \\
& \beta_{3}=\frac{1}{12}\left(2 f_{n}^{(2)}-2 n h f_{n}^{(3)}+n^{2} h^{2} f_{n}^{(4)}\right), \\
& \beta_{4}=\frac{1}{24}\left(f_{n}^{(3)}-n h f_{n}^{(4)}\right), \\
& \beta_{5}=\frac{1}{120} f_{n}^{(4)}
\end{aligned}
$$

respectively. Since one-step numerical method shall be derived, let

$$
y_{n+1}-y_{n} \equiv F\left(x_{n+1}\right)-F\left(x_{n}\right) .
$$

Substituting (20), (23)-(32) into (33), yields

$$
\begin{aligned}
& y_{n+1}-y_{n}=\frac{h}{24}\left(24 f_{n}-24 n h f_{n}^{(1)}+12 n^{2} h^{2} f_{n}^{(2)}-4 n^{3} h^{3} f_{n}^{(3)}+n^{4} h^{4} f_{n}^{(4)}\right) \\
& +\frac{h^{2}}{12}\left(6 f_{n}^{(1)}-6 n h f_{n}^{(2)}+3 n^{2} h^{2} f_{n}^{(3)}-n^{3} h^{3} f_{n}^{(4)}\right)(2 n+1)+\frac{h^{3}}{12}\left(2 f_{n}^{(2)}-2 n h f_{n}^{(3)}+n^{2} h^{2} f_{n}^{(4)}\right)\left(3 n^{2}+3 n+1\right) \\
& +\frac{h^{4}}{24}\left(f_{n}^{(3)}-n h f_{n}^{(4)}\right)\left(4 n^{3}+6 n^{2}+4 n+1\right)+\frac{h^{5}}{120} f_{n}^{(4)}\left(5 n^{4}+10 n^{3}+10 n^{2}+5 n+1\right)
\end{aligned}
$$


Setting

$$
\begin{aligned}
& S_{1}=\frac{1}{2}\left(24 f_{n}-24 n h f_{n}^{(1)}+12 n^{2} h^{2} f_{n}^{(2)}-4 n^{3} h^{3} f_{n}^{(3)}+n^{4} h^{4} f_{n}^{(4)}\right), \\
& S_{2}=h\left(6 f_{n}^{(1)}-6 n h f_{n}^{(2)}+3 n^{2} h^{2} f_{n}^{(3)}-n^{3} h^{3} f_{n}^{(4)}\right)(2 n+1), \\
& S_{3}=h^{2}\left(2 f_{n}^{(2)}-2 n h f_{n}^{(3)}+n^{2} h^{2} f_{n}^{(4)}\right)\left(3 n^{2}+3 n+1\right), \\
& S_{4}=\frac{h^{3}}{2}\left(f_{n}^{(3)}-n h f_{n}^{(4)}\right)\left(4 n^{3}+6 n^{2}+4 n+1\right), \\
& S_{5}=\frac{h^{4}}{10} f_{n}^{(4)}\left(5 n^{4}+10 n^{3}+10 n^{2}+5 n+1\right) .
\end{aligned}
$$

Therefore,

$$
y_{n+1}=y_{n}+\frac{h}{12}\left(S_{1}+S_{2}+S_{3}+S_{4}+S_{5}\right) .
$$

Equation (40) is the newly developed one step scheme.

\section{Order of accuracy of the new scheme}

According to [18], local truncation error measures the order of accuracy of any numerical method. Consider the Taylor's series expansion of the form

$$
\begin{aligned}
y\left(x_{n}+h\right)= & y\left(x_{n}\right)+h f\left(x_{n}, y\left(x_{n}\right)\right)+\frac{h^{2}}{2} f^{(1)}\left(x_{n}, y\left(x_{n}\right)\right)+\frac{h^{3}}{3 !} f^{(2)}\left(x_{n}, y\left(x_{n}\right)\right) \\
& +\frac{h^{4}}{4 !} f^{(3)}\left(x_{n}, y\left(x_{n}\right)\right)+\frac{h^{5}}{5 !} f^{(4)}\left(x_{n}, y\left(x_{n}\right)\right)+O\left(h^{6}\right) .
\end{aligned}
$$

The local truncation error for the explicit one step method is given by

$$
\tau_{n+1}=y\left(x_{n}+h\right)-y_{n+1} .
$$

Substituting (40) and (41) into (42), yields

$$
\begin{aligned}
\tau_{n+1}= & y\left(x_{n}\right)+h f\left(x_{n}, y\left(x_{n}\right)\right) \frac{h^{2}}{2} f^{(1)}\left(x_{n}, y\left(x_{n}\right)\right)+\frac{h^{3}}{3 !} f^{(2)}\left(x_{n}, y\left(x_{n}\right)\right)+\frac{h^{4}}{4 !} f^{(3)}\left(x_{n}, y\left(x_{n}\right)\right) \\
& +\frac{h^{5}}{5 !} f^{(4)}\left(x_{n}, y\left(x_{n}\right)\right)+O\left(h^{6}\right)-y_{n}-\frac{h}{4 !}\left(S_{1}+S_{2}+S_{3}+S_{4}+S_{5}\right) .
\end{aligned}
$$

Substituting (35-39) into (43), one obtains

$$
\begin{aligned}
\tau_{n+1}= & y\left(x_{n}\right)+h f\left(x_{n}, y\left(x_{n}\right)\right)+\frac{h^{2}}{2} f^{(1)}\left(x_{n}, y\left(x_{n}\right)\right)+\frac{h^{3}}{3 !} f^{(2)}\left(x_{n}, y\left(x_{n}\right)\right)+\frac{h^{4}}{4 !} f^{(3)}\left(x_{n}, y\left(x_{n}\right)\right) \\
& +\frac{h^{5}}{5 !} f^{(4)}\left(x_{n}, y\left(x_{n}\right)\right)+O\left(h^{6}\right)-y_{n}-\frac{h}{24}\left(24 f_{n}-24 n h f_{n}^{(1)}+12 n^{2} h^{2} f_{n}^{(2)}-4 n^{3} h^{3} f_{n}^{(3)}+n^{4} h^{4} f_{n}^{(4)}\right) \\
& -\frac{h^{2}}{12}\left(6 f_{n}^{(1)}-6 n h f_{n}^{(2)}+3 n^{2} h^{2} f_{n}^{(3)}-n^{3} h^{3} f_{n}^{(4)}\right)(2 n+1) \\
& -\frac{h^{3}}{12}\left(2 f_{n}^{(2)}-2 n h f_{n}^{(3)}+n^{2} h^{2} f_{n}^{(4)}\right)\left(3 n^{2}+3 n+1\right) \\
& -\frac{h^{4}}{24}\left(f_{n}^{(3)}-n h f_{n}^{(4)}\right)\left(4 n^{3}+6 n^{2}+4 n+1\right)-\frac{h^{5}}{120} f_{n}^{(4)}\left(5 n^{4}+10 n^{3}+10 n^{2}+5 n+1\right) .
\end{aligned}
$$

Solving further, (44) becomes

$$
\begin{aligned}
\tau_{n+1}= & y\left(x_{n}\right)+h f\left(x_{n}, y\left(x_{n}\right)\right)+\frac{h^{2}}{2} f^{(1)}\left(x_{n}, y\left(x_{n}\right)\right)+\frac{h^{3}}{3 !} f^{(2)}\left(x_{n}, y\left(x_{n}\right)\right)+\frac{h^{4}}{4 !} f^{(3)}\left(x_{n}, y\left(x_{n}\right)\right) \\
& +\frac{h^{5}}{5 !} f^{(4)}\left(x_{n}, y\left(x_{n}\right)\right)+O\left(h^{6}\right)-\left[y_{n}+\frac{h}{5 !}\left(120 f_{n}+60 h f_{n}^{(1)}+20 h^{2} f_{n}^{(2)}+5 h^{3} f_{n}^{(3)}+h^{4} f_{n}^{(4)}\right)\right] .
\end{aligned}
$$


By means of localizing assumption, the terms up to $h^{5}$ have been canceled, thus the local truncation of the scheme is obtained as

$$
\tau_{n+1}=O\left(h^{6}\right) .
$$

Equation (46) shows that the order of the scheme is five.

Remark 1. The local truncation error of the scheme is summarized in the following result.

Theorem 1. By means of the Taylor's series expansion and the localizing assumption, the scheme given by (40) has fifth order accuracy.

\section{Numerical experiments, discussion of results and concluding remarks}

The developed scheme was derived via the combination of two interpolants namely polynomial function and trigonometric function via MAPLE 18. The scheme (40) was implemented on biological models with the aid of MATLAB R2014a (8.3.0.532), 32-bit (Win 32) programming language.

\subsection{Numerical experiments}

Biological models that find applications in science in terms of modelling growth and decay shall be considered. The scheme (40) is implemented on these models and the results obtained were compared with the exact solutions.

Experiment 1. Assume that a colony of 100 bacteria is multiplying at the rate of $k=0.02$ per hour per individual. How many bacteria are there after 120 minutes? It is assumed that the colony grows continuously and without restriction.

Here, it is evident that the rate of population growth is proportional to the size of population. It is possible to model this exponential growth with an initial value problem of first order ordinary differential equation of the form

$$
\frac{d p}{d t}=k p, p(0)=100, k=0.02,0 \leq t \leq 120,
$$

where $p=p(t)$ is the population of the bacteria, $p(0)=p_{0}$ is the initial population of the bacteria, $t$ is the time and $k$ is the growth rate. The exact solution of (47) is obtained as

$$
p(t)=100 \exp (0.02 t)
$$
Table 1.

The comparative results analysis of the scheme (40) " $p_{n}$ " and the exact solution " $p\left(t_{n}\right)$ " with $h=10$ are shown in

Table 1. The results generated via the scheme (40) and the exact solution

\begin{tabular}{|ccccc|}
\hline$n$ & $t_{n}$ & $p_{n}$ & $p\left(t_{n}\right)$ & $e_{n}=\left|p\left(t_{n}\right)-p_{n}\right|$ \\
\hline \hline 0.00 & 0.00 & 100.0000000000 & 100.0000000000 & 0.0000000000 \\
1.00 & 10.00 & 122.1402666667 & 122.1402758160 & 0.0000091494 \\
2.00 & 20.00 & 149.1824474140 & 149.1824697641 & 0.0000223501 \\
3.00 & 30.00 & 182.2118390914 & 182.2118800391 & 0.0000409477 \\
4.00 & 40.00 & 222.5540261644 & 222.5540928492 & 0.0000666848 \\
5.00 & 50.00 & 271.8280810347 & 271.8281828459 & 0.0001018113 \\
6.00 & 60.00 & 332.0115430506 & 332.0116922737 & 0.0001492230 \\
7.00 & 70.00 & 405.5197840461 & 405.5199966845 & 0.0002126383 \\
8.00 & 80.00 & 495.3029456200 & 495.3032424395 & 0.0002968195 \\
9.00 & 90.00 & 604.9643385882 & 604.9647464413 & 0.0004078531 \\
10.00 & 100.00 & 738.9050563898 & 738.9056098931 & 0.0005535033 \\
11.00 & 110.00 & 902.5006062880 & 902.5013499434 & 0.0007436554 \\
12.00 & 120.00 & 1102.3166471884 & 1102.3176380642 & 0.0009908757 \\
\hline
\end{tabular}




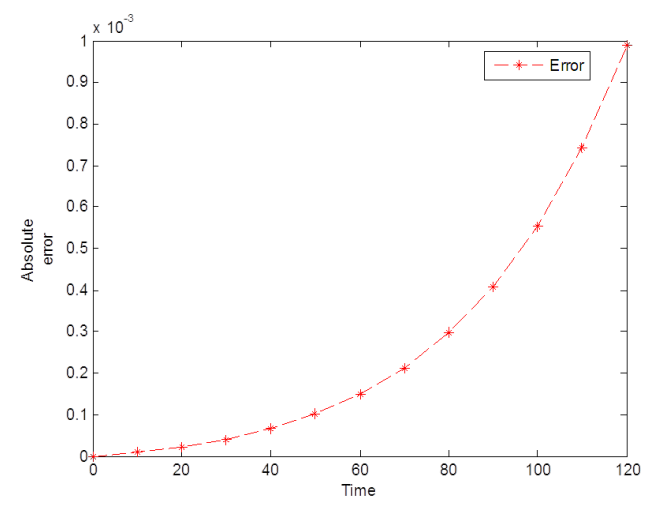

Figure 1. Errors generated via the scheme (40)

Experiment 2. Consider the exponential decay model of the form

$$
\frac{d p}{d t}=-r p, p(0)=100, r=0.02,0 \leq t \leq 120,
$$

where $p=p(t)$ is the population of the bacteria, $p(0)=p_{0}$ is the initial population of the bacteria, $t$ is the time and $r$ is the decay rate. The exact solution of (49) is obtained as

$$
p(t)=100 \exp (-0.02 t)
$$

The comparative results analysis of the scheme (40) " $p_{n}$ " and the exact solution " $p\left(t_{n}\right)$ " with $h=10$ are shown in Table 2.

Table 2. The results generated via the scheme (40) and the exact solution

\begin{tabular}{|ccccc|}
\hline$n$ & $t_{n}$ & $p_{n}$ & $p\left(t_{n}\right)$ & $e_{n}=\left|p\left(t_{n}\right)-p_{n}\right|$ \\
\hline \hline 0.00 & 0.00 & 100.0000000000 & 200.0000000000 & 0.0000000000 \\
1.00 & 10.00 & 81.8730666667 & 81.8730753078 & 0.0000086411 \\
2.00 & 20.00 & 67.0319904540 & 67.0320046036 & 0.0000141495 \\
3.00 & 30.00 & 54.8811462324 & 54.8811636094 & 0.0000173770 \\
4.00 & 40.00 & 44.9328774423 & 44.9328964117 & 0.0000189694 \\
5.00 & 50.00 & 36.7879247036 & 36.7879441171 & 0.0000194135 \\
6.00 & 60.00 & 30.1194021179 & 30.1194211912 & 0.0000190734 \\
7.00 & 70.00 & 24.6596781756 & 24.6596963942 & 0.0000182186 \\
8.00 & 80.00 & 20.1896347525 & 20.1896517995 & 0.0000170470 \\
9.00 & 90.00 & 16.5298731206 & 16.5298888222 & 0.0000157015 \\
10.00 & 100.00 & 13.5335140400 & 13.5335283237 & 0.0000142837 \\
11.00 & 110.00 & 11.0803029723 & 11.0803158362 & 0.0000128639 \\
12.00 & 120.00 & 9.0717838394 & 9.0717953289 & 0.0000114896 \\
\hline
\end{tabular}

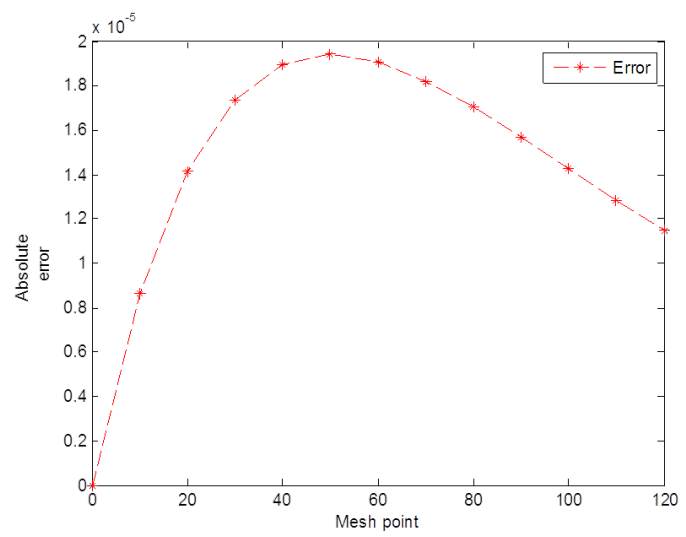

Figure 2. Errors generated via the scheme (40) 
Experiment 3. [19] Suppose there are 1000 birds on an Island, breeding with a constant continuous growth rate of $10 \%$ per year. But now birds migrate to the Island at a constant rate of 100 new arrivals per year. How many birds are on the Island after seven years?

Let $p=p(t)$ be the number of birds on Island, $t$ is the time, $k$ is a constant continuous growth rate, $m$ be the rate of migration of the population. The model equation for population growth with migration is given by

$$
\frac{d p}{d t}=k p+m, p(0)=1000, k=0.1, m=100,0 \leq t \leq 7 .
$$

The exact solution of (51) is obtained as

$$
p(t)=2000 \exp (0.1 t)-1000 .
$$

The comparative results analysis of the scheme (40) " $p_{n}$ " and the exact solution " $p\left(t_{n}\right)$ " with $h=0.5$ are shown in Table 3.

Table 3. The results generated via the scheme (40) and the exact solution

\begin{tabular}{|ccccc|}
\hline$n$ & $t_{n}$ & $p_{n}$ & $p\left(t_{n}\right)$ & $e_{n}=\left|p\left(t_{n}\right)-p_{n}\right|$ \\
\hline \hline 0.00 & 0.00 & 1000.0000000000 & 1000.0000000000 & 0.0000000000 \\
1.00 & 0.50 & 1102.5421927083 & 1102.5421927520 & 0.0000000437 \\
2.00 & 1.00 & 1210.3418360594 & 1210.3418361513 & 0.0000000919 \\
3.00 & 1.50 & 1323.6684853116 & 1323.6684854566 & 0.0000001449 \\
4.00 & 2.00 & 1442.8055161172 & 1442.8055163203 & 0.0000002032 \\
5.00 & 2.50 & 1568.0508331085 & 1568.0508333755 & 0.0000002670 \\
6.00 & 3.00 & 1699.7176148152 & 1699.7176151520 & 0.0000003368 \\
7.00 & 3.50 & 1838.1350967735 & 1838.1350971865 & 0.0000004131 \\
8.00 & 4.00 & 1983.6493947863 & 1983.6493952825 & 0.0000004963 \\
9.00 & 4.50 & 2136.6243703934 & 2136.6243709803 & 0.0000005869 \\
10.00 & 5.00 & 2297.4425407147 & 2297.4425414003 & 0.0000006856 \\
11.00 & 5.50 & 2466.5060349420 & 2466.5060357348 & 0.0000007928 \\
12.00 & 6.00 & 2644.2375998718 & 2644.2376007810 & 0.0000009092 \\
13.00 & 6.50 & 2831.0816569923 & 2831.0816580278 & 0.0000010355 \\
14.00 & 7.00 & 3027.5054137686 & 3027.5054149410 & 0.0000011723 \\
\hline
\end{tabular}

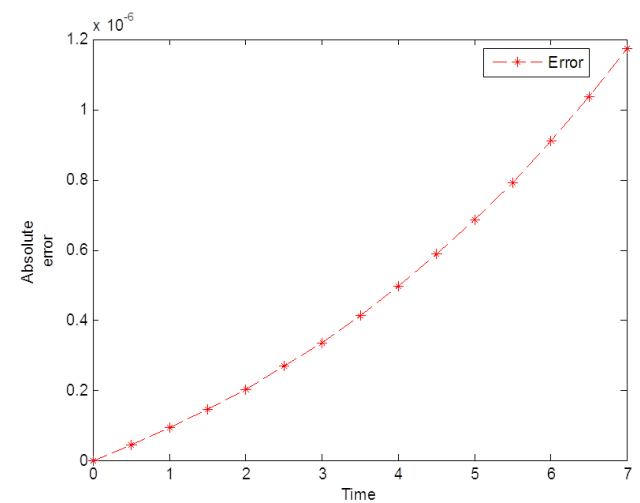

Figure 3. Errors generated via the scheme (40)

Experiment 4. [19] A cell culture in a biology laboratory currently holds 1000000 cells. The cells have a constant continuous birth rate of $1.5 \%$ and death rate of $0.5 \%$ per hour. Cells are extracted from the culture for an experiment at the rate of 5000 per hour. How many cells will be in the culture 1 hour from now?

Let $p=p(t)$ be the population, $t$ be the number of hours from now, $k$ be the difference between the birth rate and death rate, $b$ be the birth rate, $d$ be the death rate and $n$ be the extracted cells. The model equation for the growth and decay with input and output is given by

$$
\frac{d p}{d t}=(b-d) p+n, p(0)=1000000,0 \leq t \leq 1,
$$


where $b=0.015, d=-0.005, k=0.01, n=-5000$. The exact solution of (53) is obtained as

$$
p(t)=500000 \exp (0.01 t)+500000 .
$$

The comparative results analysis of the scheme (40) " $p_{n}$ " and the exact solution " $p\left(t_{n}\right)$ " with $h=0.1$ are shown in Table 4.

Table 4. The results generated via the scheme (40) and the exact solution

\begin{tabular}{|ccccc|}
\hline$n$ & $t_{n}$ & $p_{n}$ & $p\left(t_{n}\right)$ & $e_{n}=\left|p\left(t_{n}\right)-p_{n}\right|$ \\
\hline \hline 0.00 & 0.00 & 1000000.0000000000 & 1000000.0000000000 & 0.0000000000 \\
1.00 & 0.10 & 1000500.2550850213 & 1000500.2500833542 & 0.0050016671 \\
2.00 & 0.20 & 1001001.0106753386 & 1001001.0006670002 & 0.0100083384 \\
3.00 & 0.30 & 1001502.2672717074 & 1001502.2522516886 & 0.0150200188 \\
4.00 & 0.40 & 1002004.0253753845 & 1002004.0053386709 & 0.0200367136 \\
5.00 & 0.50 & 1002506.2854881278 & 1002506.2604297005 & 0.0250584274 \\
6.00 & 0.60 & 1003009.0481121978 & 1003009.0180270325 & 0.0300851653 \\
7.00 & 0.70 & 1003512.3137503569 & 1003512.2786334243 & 0.0351169326 \\
8.00 & 0.80 & 1004016.0829058710 & 1004016.0427521367 & 0.0401537343 \\
9.00 & 0.90 & 1004520.3560825090 & 1004520.3108869339 & 0.0451955751 \\
10.00 & 1.00 & 1005025.1337845444 & 1005025.0835420840 & 0.0502424604 \\
\hline
\end{tabular}

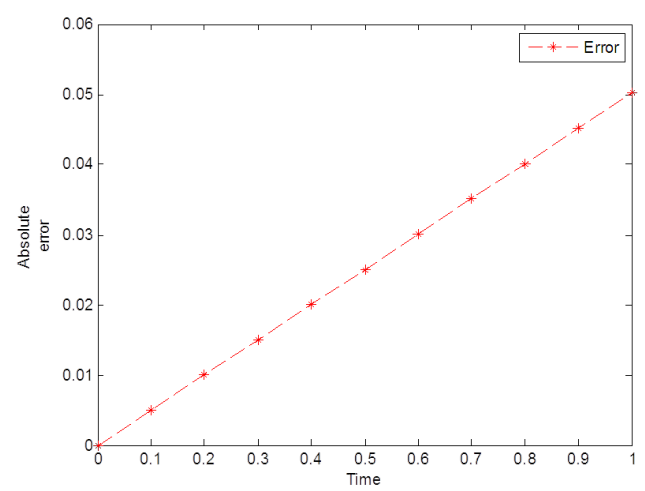

Figure 4. Errors generated via the scheme (40)

\subsection{Summary of the results}

The summary of the results generated via the scheme and the exact solution at the final mesh point time in the nearest whole number is presented as follows (Table 5):

Table 5. The summary of the accuracy of results generated via the scheme (40) against exact solution

\begin{tabular}{|ccccc|}
\hline Experiments & $t_{n f l}$ & $p_{n f l}$ & $p\left(t_{n f l}\right)$ & $e_{n f l}=\left|p\left(t_{n} f l\right)-p_{n f l}\right|$ \\
\hline \hline Experiment 1 & 120 mins. & 1102 & 1102 & 0.000 \\
Experiment 2 & 120 mins. & 9 & 9 & 0.0000 \\
Experiment 3 & 7 hours & 3027 & 3027 & 0.0000 \\
Experiment 4 & 1 hour & 1005025 & 1005025 & 0.0000 \\
\hline
\end{tabular}

\subsection{Discussion of results and concluding remarks}

In this paper, a new fifth order scheme for the solution of initial value problems in ordinary differential equations emanated from biological sciences is developed via the combination of two interpolants. Four numerical experiments have been performed to test the performance of the scheme in terms of the accuracy in the context of the exact solution as shown in Tables 1-4 and also absolute errors computed at each mesh point of the integration interval under consideration as demonstrated in Figures 1-4. The numerical results in Tables 1-4 show that the fifth order scheme is accurate and converges faster to the exact solution. The effect of the constant $k$ determines the solution of the models under consideration. It is observed from Tables 1,3 and 4 
that the results of the scheme and the exact solution increase exponentially over time. It is also observed from Table 2 that the results of both the scheme and the exact solution decrease over time. When compared with the exact solutions, the fifth order scheme yielded smaller amount of errors as seen from the above Figures 1-4. The summary of the results generated via the fifth order scheme in the context of the exact solution is presented in Table 5. Hence, the fifth order scheme is a good approach to be included in the class of linear explicit numerical methods as its analysis carried out agrees with the exact solution of exponential growth and decay models emanated biological sciences. Finally, all the calculations were carried out via MATLAB R2014a, Version: 8.3.0.552, 32 bit (Win 32) in double precision.

Conflicts of Interest: "The author declares no conflict of interest."

\section{References}

[1] Ansari, M. Y., Shaikh, A. A., \& Qureshi, S. (2018). Error bounds for a numerical scheme with reduced slope evaluations. Journal of Applied Environmental and Biological Sciences, 8(7), 67-76.

[2] Bird, J. (2017). Higher Engineering Mathematics. Taylor and Francis Group, London.

[3] Butcher, J. C. (2016). Numerical Methods for Ordinary Differential Equations. John Wiley and Sons, Ltd., United Kingdom.

[4] Jain, M. K. (2003). Numerical Methods for Scientific and Engineering Computation. New Age International (P) Ltd., New Delhi, India.

[5] Lambert, J. D. (1991). Numerical Methods for Ordinary Differential Systems: the Initial Value Problem. John Wiley and Sons, Inc., New York.

[6] Lambert, J. D. (2011). Computational Methods in Ordinary Differential Equations. John Wiley and Sons Inc.

[7] Rabiei, F., \& Ismail, F. (2011). Third-order Improved Runge-Kutta method for solving ordinary differential equation. International Journal of Applied Physics and Mathematics, 1(3), 191-194.

[8] Applications of Differential Equations. http:// faculty.bard.edu/ belk/math213s14, accessed on April 27, 2020.

[9] Zill, D. G. (2012). A First Course in Differential Equations with Modeling Applications. Cengage Learning.

[10] Qureshi, S., \& Emmanuel, F. S. (2018). Convergence of a numerical technique via interpolating function to approximate physical dynamical systems. Journal of Advanced Physics, 7(3), 446-450.

[11] Fadugba, S. E., \& Idowu, J. O. (2019). Analysis of the properties of a third order convergence numerical method derived via transcendental function of exponential form. International Journal of Applied Mathematics and Theoretical Physics, 5(4), 97-103.

[12] Ogunrinde, R. B., \& Fadugba, S. E. (2012). Development of a new scheme for the solution of initial value problems in ordinary differential equations. International Organization of Scientific Research Journal of Mathematics (IOSRJM), 2, 24-29.

[13] Fadugba, S. E., \& Okunlola, J. T. (2017). Performance measure of a new one-step numerical technique via interpolating function for the solution of initial value problem of first order differential equation. World Scientific News, 90, 77-87.

[14] Fadugba, S. E., \& Olaosebikan, T. E. (2018). Comparative study of a class of one-step methods for the numerical solution of some initial value problems in ordinary differential equations. Research Journal of Mathematics and Computer Science, 2(9), 1-11.

[15] Qureshi, S., Shaikh, A. A., \& Chandio, M. S. (2019). A new iterative integrator for Cauchy problems. Sindh University Research Journal-SURJ (Science Series), 45(3).

[16] Rabiei, F., \& Ismail, F. (2012). Fifth-order Improved Runge-Kutta method for solving ordinary differential equation. Australian Journal of Basic and Applied Sciences, 6(3), 97-105.

[17] Rabiei, F., Ismail, F., \& Suleiman, M. (2013). Improved Runge-Kutta methods for solving ordinary differential equations. Sains Malaysiana, 42(11), 1679-1687.

[18] Fadugba, S. E. \& Qureshi, S. (2019). Convergent numerical method using transcendental function of exponential type to solve continuous dynamical systems. Punjab University Journal of Mathematics, 51(10), 45-56.

[19] Brown, P., Evans, M., \& Hunt, D. (2013). Growth and decay- $A$ guide for teachers (Years 11-12). Educational Services, Australia.

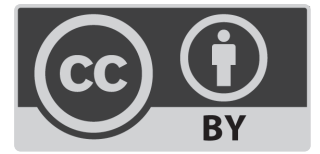

(c) 2021 by the authors; licensee PSRP, Lahore, Pakistan. This article is an open access article distributed under the terms and conditions of the Creative Commons Attribution (CC-BY) license (http://creativecommons.org/licenses/by/4.0/). 